\title{
ALMAS SENSUALES: MODOS \\ INCORPÓREOS DE SENTIR Y CONOCER EN LA AMAZONÍA INDÍGENA*
}

\section{Fernando Santos Granero}

Los yanesha de la Amazonía peruana estarían de acuerdo con Aristóteles y Santo Tomás de Aquino en que el conocimiento sólo puede ser obtenido a través de la percepción sensorial. Sin embargo, estarían en desacuerdo sobre qué exactamente significa 'percepción sensorial'. En la tradición occidental los sentidos son considerados como el modo "fisiológico" de percepción. Sólo podemos conocer, se dice, a través del cuerpo y sus sentidos: vista, oído, olfato, tacto y gusto. En contraste, los Yanesha consideran los sentidos corporales como medios imperfectos de conocimiento, incapaces de percibir la verdadera dimensión espiritual del mundo. Sólo uno de los componentes incorpóreos del ser, yecamquërñ o 'nuestra vitalidad', está dotado de las facultades sensoriales que permiten tener una percepción correcta y, con ello, la posibilidad de obtener un conocimiento 'verdadero'. Es por esta razón que, desde el punto de vista yanesha, las vitalidades son sensuales mientras que los cuerpos son en cierta medida insensibles. Este artículo explora los modos incorpóreos de percepción y conocimiento de los Yanesha, así como sus teorías de percepción y su jerarquía de los sentidos. El objetivo es abogar por una renovada antropología de los sentidos en los estudios amazónicos, así como proponer una revisión crítica de la noción de 'perspectivismo'.

Yanesha people of eastern Peru would agree with Aristotle and St. Thomas Aquinas in that knowledge can only be achieved through sense perception. They would, however, disagree on what exactly 'sense perception' means. In the Western tradition the senses are considered to be the 'physiological' modes of perception. We can only know, it is asserted, through the body and its senses: sight, hearing, smell, touch, and taste. In contrast, yanesha people view bodily senses as imperfect means of knowing, unable to grasp the true, spiritual dimension of the world. Only one of the non-corporeal components of the self, yecamquërn or 'our vitality', is endowed with the sensory faculties that allow for a correct perception, and thus for the possibility of 'true' knowledge. It is for this reason that, from a yanesha point of view, vitalities are sensual, whereas bodies are considered to be somewhat insensible. This article explores yanesha non-corporeal modes of sensing and knowing, as well as their theories of perception and sensual hierarchies. My purpose is to advocate for a renewed anthropology of the senses in Amazonian studies, as well as to propose a critical revision of the notion of Amerindian perspectivism

Originalmente publicado en el Volumen especial de Tipiti en homenaje a Joanna Overing (en prensa) Traducido del inglés por Luisa Elvira Belaunde. 
Tuve mi primera lección sobre la teoría yanesha de la percepción mientras pescaba en el río Palcazu, una noche oscura de mayo de 1977, junto con Matar, jefe tradicional de Camantarmas y mi auto-proclamado mentor. Era por entonces un estudiante de licenciatura haciendo trabajo de campo por primera vez entre los yanesha de la Selva Central y había estado en el área alrededor de dos meses. Cansado de mis muchas preguntas y del hecho de que constantemente enredaba mi línea de pescar con la suya, Matar me sugirió discretamente que me fuese a sentar un poco más lejos, donde, me aseguró con gran entusiasmo, había un fantástico sitio para pescar. Mientras estaba ahí, solo, mascando hojas de coca y fumando sin parar para disipar el aburrimiento, comencé a escuchar gente cantando una hermosa canción. Era una canción sherareñets perteneciente al estilo femenino de cantos sagrados coshaminats. Las voces de las cantantes estaban acompañadas de música de carrizo o flauta de pan, conocida como requërqueñets; sus voces soprano se fundían suavemente con los sonidos agudos de las dos flautas principales y el tono más grave de las flautas grandes que las acompañaban.

Aunque aún tenía dificultades para orientarme en las muchas curvas y vueltas del río Palcazu, estaba seguro que Shecor, el hermano mayor de Matar, vivía justo en la orilia del frente de donde estábamos pescando. Mientras escuchaba cautivado la música, me acordé que la hija de Shecor había sido ritualmente recluida hacía cinco semanas tras tener su primera menstruación. Debía salir de su reclusión la próxima luna llena. Sus padres me habían dicho que querían que yo estuviera presente en su fiesta de 'salida'. Por esa razón me extrañaba que hubieran decidido hacer la fiesta antes de lo planeado y me sentía un poco desilusionado por no haber sido invitado.

Después de escuchar la música por un rato, decidí preguntarle a Matar si es que sabía algo sobre la fiesta. Cuando lo hice, me miró desconcertado. ¿"Qué fiesta?" - me preguntó. "La de la casa de Shecor al otro lado del río" - le contesté; “¿Ne escuchas la música?”. Con una expresión aun más extrañada y alarmada, Matar me dijo que al otro lado del río no vivía nadie y que él no escuchaba música alguna Pensé que estaba haciendo como que no escuchaba la música para evitarme la mortificación de no haber sido invitado a la fiesta, pero sus acciones posteriore enseguida me demostraron que no estaba fingiendo. Me dijo que guardara mi líne: de pescar rápidamente y me preparara para partir de inmediato. Cuando le pregun: por qué, me rogó que me quedase callado. Después de juntar nuestras cosas manera apresurada, saltamos a la canoa y en absoluto silencio nos deslizamos tí abajo hasta llegar al puerto de su casa.

Solamente cuando nos encontramosa salvo en su casa, sentados junto al fog y mascando un nuevo puñado de hojas de coca, me dijo Matar que lo que ha: escuchado no eran voces humanas sino el canto de Pocoy, una clase de seres s acuáticos que los Yanesha llaman sirenas en castellano. Escuchar a Pocoy, me da 
nno es solo raro sino muy peligroso. Normalmente imperceptibles durante el día, las sirenas solo se revelan a los pescadores solitarios. Lo hacen para seducirlos. Si el zescador incauto escucha las canciones seductoras de Pocoy y la llama, ésta se aparece bajo la forma de una hermosa mujer rubia. Dichos encuentros son, por lo general, fatales. Bellamente pintada, perfumada con semillas fragantes, vestida con túnicas orillantes, hablando con un tono de voz acariciante, dulce de probar, y suave de tocar, Pocoy se apodera de los sentidos del hombre al que escoge. Envuelto por sus encantos, el hombre pierde su poder de voluntad y cae bajo el hechizo de Pocoy, cuyo único deseo es llevárselo para convertirlo en su amante en su mundo sub-acuático.

Es por esta razón que Matar estaba tan ansioso por irse. Si no me hubiese ido mientras aún tenía la voluntad para hacerlo, me explicó, podría haber sentido la tentación de comunicarme con la sirena. Y si lo hubiese hecho, nunca hubiera podido regresar a mi casa. No sé exactamente qué fue lo que pasó esa noche. ¿Realmente escuché a una sirena? ¿Tuve una alucinación acústica? ¿O todo lo que pasó no fue más que un engaño de mis sentidos? Sea como fuere, esa noche aprendí mucho sobre cómo los yanesha 'conciben' el mundo y, sobre todo, sobre cómo 'perciben' el mundo. En los meses siguientes habría de aprender mucho más.

En este artículo presento los principios en los que se sustenta la teoría yanesha de la percepción y el conocimiento y discusión como dicha teoría contrasta de maneras importantes con la nuestra. Mi propósito es abogar por una renovada antropología de los sentidos en los estudios amazónicos (Véase Howes 1991, 2005), así como proponer una revisión crítica de la noción de 'perspectivismo'. Al hacer esto, me adhiero a lo que considero es uno de los argumentos más poderosos de Joanna Overing (1985) en su obra Reason and Morality (Razón y moralidad). En este texto la autora sostiene que "La humildad se alcanza únicamente mediante la familiaridad con las epistemologías y las ontologías de otras culturas, una familiaridad conseguida por medio de la rica etnografía que se obtiene cuando se toma seriamente en cuenta lo que otros dicen sobre sus mundos sociales". Desde entonces me he regido por este precepto.

\section{El ser y sus componentes}

Las ideas yanesha sobre el ser y sus sentidos son mucho más complejas que las que prevalecen en las sociedades occidentales. Desde su punto de vista, todo individuo está compuesto de un cuerpo y dos entidades incorpóreas: yecamqquëri ('nuestra alma/vitalidad'), y yechoyeshem ('nuestra sombra'). El cuerpo es concebido como la dimensión material del ser. El término chetsots, que significa tanto 'carne' como 'cuerpo', pone énfasis en la cruda materialidad del cuerpo. A otro nivel, sin embargo, los yanesha conciben el cuerpo como una túnica que envuelve sus elementos no-corporales. Esta concepción alude a las largas túnicas de algodón cashemuets (fem.), shetamulets (masc.)- que los yanesha usaban a diario hasta hace poco y que aún usan en la intimidad de sus casas o en ocasiones formales. Esto no es 
sorprendente, ya que envueltos en sus túnicas, los hombres y mujeres yanesha se conviertan en sus túnicas (ver figura 1).

En contraste con lo que Viveiros de Castro (1998: 471) sugiere para los pueblos amerindios en general, según los yanesha la relación entre cuerpos y túnicas no es metafórica sino literal: los cuerpos son túnicas y las túnicas son cuerpos. Esto es confirmado por las narraciones míticas y las creencias chamánicas. Al final de los tiempos míticos, los humanos retuvieron sus túnicas -es decir, sus cuerpos/túnicas humanos- mientras que las túnicas decoradas de los animales, los espíritus y los otros espíritus fueron transformadas en sus cuerpos actuales. Así, se dice que las placas del armadillo, las manchas del jaguar y la negrura del paujil derivan de los diseños y colores de sus túnicas míticas. Es más, los yanesha aseguran que cuando los chamanes consumen narcóticos o sustancias alucinógenas, sus almas/vitalidades viajan a otros planos del mundo, dejando sus 'túnicas' -nuevamente, en el sentido tanto de cuerpo como de túnica- atrás. Si sus almas son atrapadas o devoradas mientras se encuentran viajando por estos sitios, se cree que sus cuerpos se marchitan y el chamán muere.

Los yanesha consideran al cuerpo y sus sentidos como importantes fuentes de información y medios de comunicación con el mundo material. Sin embargo, piensan que los sentidos corporales son incapaces de percibir la dimensión espiritual, normalmente escondida, que subyace al mundo visible. Ni siquiera los chamanes pueden percibir la dimensión espiritual de las cosas mediante sus sentidos corporales (Véase Viveiros de Castro 2004b: 465 y Vilaça 1992: 66, 81, para nociones diferentes

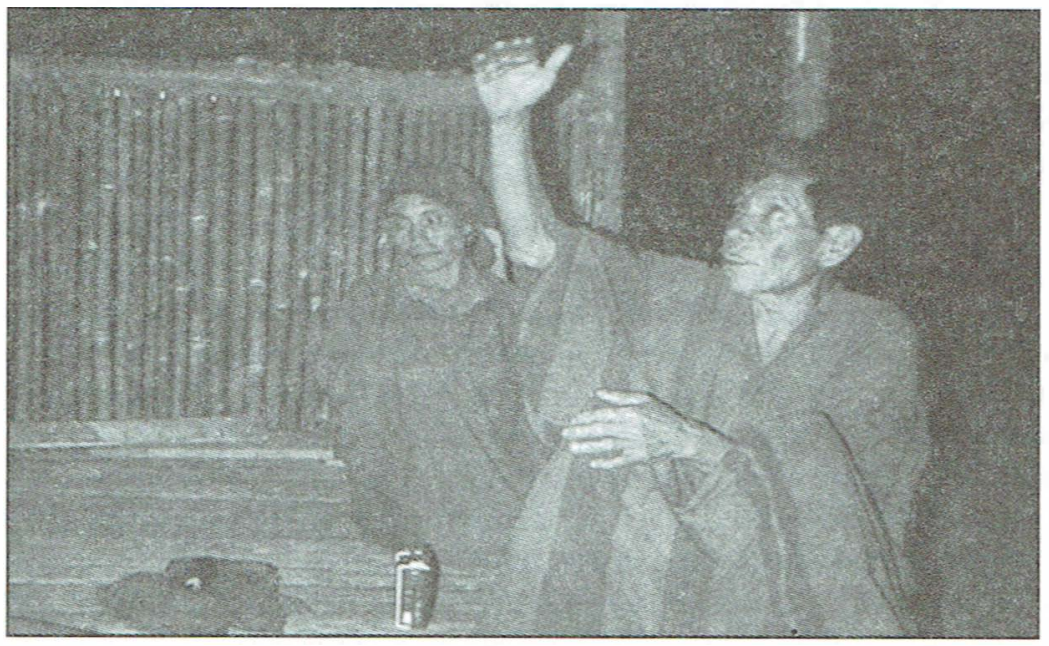

Figura 1:

Los cuerpos son túnicas y las túnicas son cuerpos 
entre otros pueblos amerindios). Estos sentidos son, por lo tanto, concebidos como medios inadecuados para adquirir/producir conocimientos significativos. Esta tarea solo puede ser llevada a cabo por los componentes incorpóreos de una persona. No obstante, los yanesha afirman que existen importantes diferencias entre estos componentes en términos de su conexión con el cuerpo, sus capacidades sensoriales y su habilidad para la producción de conocimiento.

Se dice que yechoyeshem, 'nuestra sombra,' está permanentemente conectada al cuerpo mientras la persona de la que es parte se encuentra con vida. Su manifestación visible, tal como lo indica su nombre, es la sombra de una persona. Al igual que las sombras, las cuales son totalmente dependientes de los cuerpos de los que son una proyección, yechoyeshem está privada de conciencia propia, voluntad y sentidos. Como tal, es incapaz de percibir el mundo y generar conocimiento. Solamente al morir la persona de la que son parte, las sombras se liberan de la tiranía del cuerpo; y solamente entonces adquieren voluntad propia y algunas de las capacidades sensoriales de los cuerpos a los que habían estado conectadas. Llamadas choyeshemats, o 'sombras errantes', estos ex-componentes de seres humanos se quedan a veces cerca de donde solía vivir o donde murió el finado, penando y aterrorizando a los vivos.

En cambio, se dice que yecamquërin, un término que a menudo los yanesha traducen al castellano como 'nuestra alma', está dotada de las mismas capacidades sensoriales del cuerpo. De hecho, los yanesha consideran que es el alma/vitalidad la que confiere facultades sensoriales al cuerpo. Afirman que yecamquëri es la manifestación individual del alma de las divinidades creadoras de las categorías yato '('nuestro abuelo') y yompor ('nuestro padre'). Conocida como camuequeñets, esta alma genérica es concebida como la fuente primordial de toda vida en el cosmos. Por lo tanto, yecamquërĩ es concebida como la manifestación humana individualizada de la fuerza de vida que los dioses creadores comparten con todos los seres vivos. Es esta dimensión incorpórea del ser la que infunde vida al cuerpo y le permite tener tanto una 'vida de los sentidos' como una 'vida de los pensamientos' (Overing 2006). Sin ella, los cuerpos no serían más que pura materia despojada de vida al igual que las túnicas a las que son equiparados. En la medida en que los yanesha aseguran que camuequeñets, el alma genérica divina, está compuesta de aliento/fuerza vital, la mejor traducción del término yecamquëñ es 'nuestra vitalidad' más que 'nuestra alma'.

Yecamquëri tiene dos manifestaciones. La primera, la vitalidad propiamente, es capaz de desprenderse del cuerpo y vagar por éste y otros niveles del multiverso. Tiene la misma forma y fisonomía de la persona de la que hace parte y, en este sentido, se puede decir que desde un punto de vista yanesha, la 'persona' y sus componentes tienen una naturaleza 'fractal' (Luciani Kelly 2001). Sin embargo, en contraste con otros pueblos amazónicos, los yanesha sostienen que las vitalidades 
están hechas de aliento/fuerza divina y por lo tanto carecen de corporalidad. ${ }^{1}$ Por esta razón, las vitalidades no tienen límites fijos y pueden difundirse hacia los objetos que están en contacto prolongado con un individuo, como sus adornos. En contraste, la segunda manifestación de yecamquëñ es inseparable del cuerpo. Bajo la forma de un pequeño homúnculo, este componente del ser se ubica en las pupilas de la persona de la que hace parte. Cuando nuestra vitalidad se desprende para ir a pasear por el mundo, la parte no separable - llamada pacheñmer, 'su ser-como-gente'- se queda cuidando el cuerpo, el cual, privado de su fuente de vida, es vulnerable a todo tipo de accidentes y ataques sobrenaturales.

Al igual que el cuerpo, nuestra vitalidad está dotada de capacidades sensoriales. Pero según los Yanesha, mientras los sentidos del cuerpo sólo perciben la túnica de las cosas, es decir, su apariencia material, los sentidos no-corporales de nuestras vitalidades son capaces de percibir las cosas como 'realmente son'. En otras palabras, pueden percibir la dimensión espiritual de las cosas, lo cual, en términos perspectivistas, es lo mismo que decir que pueden percibir a los animales, espíritus y otros seres no-humanos como estos seres se perciben a sí mismos, es decir, como seres humanos. Cabe notar que en contraste con otros pueblos amerindios (Viveiros de Castro 2004b: 468), los yanesha le atribuyen esta capacidad a todos los seres humanos y no solamente a los chamanes. Reconocen tres circunstancias en que nuestras vitalidades se encuentran libres para ejercer sus capacidades sensuales y por lo tanto involucrarse en procesos cognitivos: 1. cuando la gente duerme; 2. durante vigilias rituales; y 3 . tras consumir narcóticos o alucinógenos. Desde una perspectiva occidental, bajo estas circunstancias los sentidoscorporales se encuentran disminuidos, atontados o sobre-estimulados. Desde una perspectiva yanesha, estos sentidos son simplemente dejados atrás, junto con el cuerpo, permitiendo así la activación de los sentidos de las vitalidades desencarnadas o desencarnadas. Debe notarse, por lo tanto, que la teoría yanesha de la percepción no está construida sobre la base de una oposición entre 'cuerpo' y 'vitalidad', sino más bien sobre la base del contraste entre las facultades sensoriales de las vitalidades encarnadas y desencarnadas $\multimap$, tal como lo pondría Stolze Lima (2000: 48), "entre la realidad del sujeto y la realidad de su alma." Desde este punto de vista, las almas/vitalidades son las que dotan a los individuos con la capacidad para llevar una vida sensual. Mientras que las vitalidades encarnadas solo pueden percibir la túnica de las cosas, las vitalidades desencarnadas pueden percibir su dimensión espiritual de forma humana.

Al igual que Wittgenstein (1974), algunos autores (Viveiros de Castro 1998; Stolze Lima 1999: 122 conciben a las almas no como entidades inmateriales, sino como seres humanos. Desde este punt: de vista, la equivalencia entre cuerpo y alma -en la medida que el cuerpo humano es visto como una suerte de fotografía del alma humana- le confiere a las almas cierto grado de corporalidaz. Aunque los yanesha estarían de acuerdo en que las almas no son inmateriales, los mismos trazan una clara distinción entre la materialidad cruda y limitada de chetsots (cuerpo-en-tanto-carne) y la materialidad ligera y difusa de yecamquëm (vitalidad-como-aliento/fuerza). Es por ello que niegan a esta última un carácter 'corporal'. 


\section{El cuerpo y sus sentidos}

Al igual que el occidental promedio, los yanesha consideran que el cuerpo está dotado de cinco sentidos básicos: el oído (e'mueñets), la vista (enteñets), el olfato (mosyeñets), el tacto (a'plleñets), y el gusto (amlleñets). Todos estos sentidos son considerados indispensables para recolectar el tipo de información factual necesaria para vivir en esta tierra. No a todos, sin embargo, se les atribuye la misma importancia. Al igual que los occidentales, los yanesha consideran que la vista y el oído son los sentidos más importantes. Pero, en contraste con ellos y al igual que muchos otros pueblos amerindios, le atribuyen un mayor significado al oído que a la vista (ver Seeger 1981; Passes 1998, 2001, Murphy 2004). Esto confirma la proposición de McLuhan (1961) según la cual las sociedades con escritura privilegian la vista y lo visual, mientras que las sociedades sin escritura tienden a favorecer el oído y lo auditivo. Aunque este argumento ha sido recientemente cuestionado por Classen (2005), queda claro que los pueblos sin escritura frecuentemente privilegian otros sentidos que la vista como el principal medio de adquisición de conocimiento.

Nunca estuve más conciente de la importancia del oído que en una ocasión en 1983, durante mi segundo trabajo de campo entre los yanesha, cuando invité a Mañor a Lima para ayudarme a transcribir y traducir un número de mitos que había grabado en el campo. Era la hora del atardecer y estábamos trabajando juntos en la sala cuando vimos una rata entrar en la casa desde el jardín. Corrimos tras ella pero no pudimos ver en que cuarto se había metido. Revisamos todos los cuartos y el baño, miramos debajo de todas las camas y detrás de todos los muebles, pero sin éxito. Fue entonces que Mañor dijo que él podía encontrarla, pero que necesitaba silencio absoluto. Entró al primer cuarto, apagó la luz, cerró la puerta y se sentó en silencio en la oscuridad. Después de unos diez minutos salió, diciendo que la rata no estaba ahí. Hizo lo mismo en el segundo cuarto y con el mismo resultado. Después de cinco minutos en el tercer cuarto, sin embargo, salió y declaró que la rata estaba ahí, dentro del cajón superior del armario. Cuando le pregunté cómo había sabido dónde estaba, me dijo que al entrar al cuarto la rata lo escuchó y se quedó quieta. Pero al cabo de unos minutos de silencio total, la rata se sintió a salvo y comenzó a moverse. El ruido casi imperceptible de sus patas contra el fondo del cajón traicionó su escondite.

La vista es el segundo sentido en orden de importancia para los yanesha. Los cazadores y pescadores tienen una visión increíblemente aguda tanto de día como de noche. Cuando cazan de día, pueden distinguir un paujil, un mono y hasta un perezoso moviéndose lentamente a lo largo de una rama, allí donde para mí no había sino una masa verde informe. Cuando pescan en la tenue luz de la madrugada no tienen problema en ajustar su vista a la refracción del agua y pueden traspasar un pescado con sus flechas de tres puntas a una distancia de hasta dos metros. Y cuando cazan de noche, sentados en el bosque o navegando a lo largo del río en una 
canoa, tienen poca dificultad en identificar con la luz de sus linternas los ojos brillantes de animales que se encuentran hasta a veinte metros de distancia.

El tercer sentido en importancia es el olfato. Este sentido proporciona información útil tanto para la caza como para llevar a cabo diversas actividades de subsistencia. Los cazadores yanesha tienen un fino sentido del olfato. Cuando están caminando por el bosque pueden oler si es que hay palmeras con frutos maduros en la vecindad, o si ciertos árboles productores de resinas se encuentran en las cercanías. Más importante aún, pueden detectar si es que un determinado animal ha pasado por el camino y hace cuanto tiempo. Matar aseguraba que era especialmente bueno en identificar a los animales por su olor. Un día que estábamos caminando por el bosque en busca de cierta liana tuve prueba de su capacidad. En un determinado momento Matar se detuvo abruptamente y me hizo señas para que hiciera lo misma Olió el aire y me dijo que un jaguar había cruzado el camino hacía poco. Unos metro: más adelante encontramos las huellas de un jaguar, frescamente impresas en el suel arcilloso de la trocha.

Los yanesha no son tan claros acerca del orden de importancia de los otros dos sentidos. El tacto es crucial para algunas actividades, tales como pesca carachamas. Como las carachamas se alimentan chupando micro-algas, siempre encuentran pegadas a los cantos rodados del fondo del río. La gente que quie pescar carachamas deambula por la parte menos profunda del río sintiendo con $\Sigma_{-}$ manos las piedras del fondo, donde éstas suelen esconderse. La vista no es de much ayuda en esta actividad. Todo depende del tacto. Cuando uno siente las durz escamas de la carachama debe atraparla rápidamente antes que la misma se esc: entre las manos.

El gusto es quizás el sentido de menor importancia para recoger informa del mundo natural. Proporciona, sin embargo, datos relevantes para llevar a ciertas actividades. En el pasado, cuando las mujeres yanesha iban en busca de and para sus ollas solían probar y oler los diferentes tipos de arcilla que encontra:para determinar cual era la más adecuada. Al igual que entre los matsigenka (She 2004) y los bororo (Crocker 1985: 160), entre los yanesha el gusto y el olfato tamson cruciales para identificar ciertas plantas, arbustos y hierbas de import medicinal o mágica.

A unque los yanesha consideran que los sentidos corporales son indisper para la praxis humana en esta tierra, tienen poca confianza en su capacida aprehender lo que consideran es la verdadera naturaleza del mundo y sus igual que los cashinahua (Kensinger 1995: 237-246; Lagrou 2000: 157) y otros amerindios que sostienen que el conocimiento reside en el cuerpo, los consideran que el conocimiento/memoria reside en el corazón (ver Rosengr y Belaunde 2006, para ideas similares entre los matsigenka y Airo-Pai). Sin er son claros en cuanto a que los pensamientos (cotapñats) no son producidos 
cuerpo, sino por el alma/vitalidad de un individuo. La noción amerindia según la cual el mundo material es la envoltura de una dimensión espiritual que es la fuente de conocimiento extraordinario parece ser tan universal en la América tropical como la idea de que esta dimensión espiritual sólo puede ser percibida por el alma o la vitalidad de una persona. Hasta los pueblos amerindios que sostienen que el conocimiento es siempre un conocimiento encarnado o corporizado, concuerdan en que la dimensión espiritual del mundo sólo puede ser aprehendida por los componentes incorpóreos del ser. "Para ver la verdadera naturaleza de la gente y de las cosas que constituyen el mundo natural -dice Kensinger (1995: 240) con respecto a los cashinahua- uno debe comprender el bedu yushin, o espíritu del ojo, el cual también es conocido como yushin kuin, o espíritu verdadero." Lo que vemos, oímos, gustamos, olemos y tocamos mientras estamos despiertos son solamente las túnicas -tal como afirmarían los yanesha, cashinahua y otros pueblos amerindios- de los seres vivos y objetos materiales que pueblan esta tierra. Estos otros seres y objetos tienen vitalidades que sólo pueden ser percibidas por la vitalidad de una persona bajo circunstancias específicas. Dichas vitalidades tienen forma corporal -siempre humana- e incluso capacidades sensoriales pero, por lo menos desde el punto de vista yanesha, carecen de corporalidad.

\section{Teorías de la Percepción}

Los yanesha están más cerca de Platón que de Aristóteles en cuanto a sus ideas sobre cómo se produce el conocimiento. Según la filosofía aristotélica, la percepción sensual constituye la base de todo conocimiento. El mundo físico está constituido por objetos compuestos de materia -el material del que están hechos-y forma -su figura. Por medio de los sentidos, que perciben la forma de los objetos materiales, los individuos recogen información sobre el mundo. Según Aristóteles, bajo la forma de preceptos, esta información sensorial fluye por el sistema sanguíneo hasta el sensus communis, la facultad perceptiva primaria. Dicho órgano está ubicado en la región del corazón y su principal función sería la de discriminar entre los preceptos recibidos de los diversos órganos sensoriales. Con la ayuda del intelecto, que según Aristóteles es la facultad que nos permite conocer, comprender y pensar, esta información es juzgada e interpretada. Según esta visión, la cual, con ligeros cambios, constituye la base del pensamiento científico actual, el conocimiento es el producto de la información sensorial procesada por el intelecto o la razón.

En contraste, Platón distingueclaramente entre el 'mundo sensible' -el mundo de la percepción- y lo que él denomina el 'mundo inteligible' -el mundo de las formas abstractas. Caracterizado por estar en permanente flujo y cambio, el mundo sensible es un mundo de apariencias. Platón sostiene que el conocimiento debe tener por objeto lo genuinamente real y no las apariencias. Afirma que el conocimiento significativo no puede ser obtenido mediante la aprehensión sensorial del mundo físico. Por el contrario, sólo puede ser obtenido mediante el uso de la razón para comprender las formas o sustancias eternas que constituyen el mundo verdadero. 
Estas entidades abstractas existen independientemente del mundo sensible. Los objetos ordinarios, imperfectos y siempre cambiantes son copias distorsionadas de sus formas perfectas e inmutables. Puesto que tenemos una noción de formas abstractas tales como 'naranja', 'mesa', o 'justicia', y puesto que los sentidos corporales son medios imperfectos de conocimiento de estas formas, Platón sostiene que la percepción del mundo real es sólo posible por medio del alma.

La semejanza entre las teorías yanesha y platónicas del conocimiento termina, sin embargo, aquí. Tal como lo ha señalado Descola (1996: 375), uno debe desconfiar del tipo de platonismo ingenuo que con frecuencia se atribuye a los pueblos amerindios. Platón propone que nacemos con una noción de las formas abstractas que constituyen el mundo real. Puesto que los sentidos corporales son incapaces de percibir estas formas, concluye, primeramente, que el alma precede al cuerpo, es decir, que debe de ser eterna e inmortal, y en segundo lugar, que el conocimiento precede a la existencia del cuerpo y sólo puede ser aprehendido como la memoria de una vida pasada.

Los yanesha también creen que yecamquërin, nuestra alma / vitalidad, es eterna e inmortal y que todo conocimiento significativo sólo puede ser obtenido por intermedio de su agencia. Sin embargo, dado que consideran que las vitalidades son meras manifestaciones de camulequeinets, el aliento/fuerza de los dioses creadores, y no entidades discretas e incambiables, no les atribuyen la capacidad de pasar de un individuo muerto a un recién nacido, o la posibilidad de transmitir conocimiento del uno al otro. Por el contrario, consideran que las divinidades creadoras infunden vitalidad en cada recién nacido y que esta vitalidad regresa a su fuente divina -ubicada en Yomporesho, la residencia celestial de los dioses creadores- al morir la persona. De esta manera, las vitalidades están en constante circulación y transformación, pasando de un estado genérico divino a una forma individualizada humana y nuevamente a un estado genérico. El conocimiento, desde este punto de vista, no puede ser una memoria del pasado. Puede ser transmitido de una persona a otra por medio de la enseñanza y el aprendizaje, pero la producción de nuevos conocimientos es siempre una proeza individual. Dicha producción es sólo posible a través de las actividades del alma/vitalidad de un individuo y, a menos que sea transmitido mediante la enseñanza, el conocimiento así adquirido se pierde al morir la persona.

\section{La producción de conocimiento}

Desde la perspectiva yanesha, el conocimiento (eñoteñets) es siempre un conocimiento extraordinario que tiene su origen en los seres que habitan otros niveles del multi-verso. Si bien es cierto que los yanesha han acumulado una impresionante cantidad de información sobre un amplio rango de campos de conocimiento agricultura y astronomía, botánica y zoología, hidrología y meteorología, navegación y arquitectura, medicina y herboristería- y si bien es cierto que esto es el resultado de siglos de acumulación de conocimiento empírico por innumerables generaciones 
de yanesha, si uno les pregunta como se originó este conocimiento, a nadie se le ocurre mencionar la observación, registro e interpretación paciente y sistemática de hechos naturales. Tampoco lo atribuirían a la experimentación por ensayo y error y la acumulación colectiva de sus resultados a lo largo del tiempo. El verdadero conocimiento, según los pensadores yanesha, siempre proviene de otros niveles del mundo. Esto es válido incluso en lo que concierne al conocimiento de actividades tan básicas como el cultivo de la yuca, el cual fue obtenido de la Paca, el uso del barbasco, derivado del semen del gigante Hua'tenañ, o el procesamiento de la sal y el hierro, aprendidos de los generosos Posona' y Asreso.

Este tipo de conocimiento fue conferido a los yanesha en tanto colectividad en tiempos míticos. Actualmente, sin embargo, el conocimiento extraordinario es adquirido, ya sea directamente a través de un esfuerzo personal, o indirectamente a través de gente sabia, por lo general personas mayores. En ambos casos, el proceso de aprendizaje es considerado como una búsqueda ritual de conocimiento en la que el que busca debe prepararse tanto física como espiritualmente. Esto es así por cuanto se cree, primero, que la adquisición de conocimiento extraordinario sólo puede ser lograda mediante la agencia de la vitalidad de una persona, y segundo, que la adquisición de este tipo de conocimiento es una tarea peligrosa y, por lo tanto, debe estar acompañada de formas crecientes de auto-control (ver Overing 1975, SantosGranero 1991 y Gow 2006, sobre los peligros del exceso de conocimiento).

Los sueños son una fuente importante de conocimiento, que está disponible para todos, hombres y mujeres, ancianos y jóvenes. Los yanesha aseguran que cuando dormimos nuestras vitalidades se desprenden de nuestros cuerpos y se pasean por éste y otros niveles de mundo. Nuestros sueños reflejan el deambular de nuestras vitalidades y sus encuentros con otros seres, normalmente imperceptibles: las vitalidades de otros seres humanos, las sombras de los difuntos, las almas primordiales de los animales, las plantas y los objetos, o una vasta gama de seres espirituales y divinidades. Sin embargo, los sueños pueden ser engañosos y el conocimiento que uno puede obtener por medio de ellos es sumamente azaroso. Las vitalidades desencarnadas sólo pueden recoger fragmentos aleatorios de conocimiento durante sus visitas a otros niveles de mundo. A menos que la persona se entrene a sí misma para tener 'sueños lúcidos', es decir, para tener plena conciencia durante el sueño y así poder alterar de manera intencional el curso de los eventos en los sueños (ver Santos-Granero 2003), sus vitalidades son incapaces de producir conocimiento; sólo pueden ser receptores pasivos de conocimiento.

A fin de obtener nuevos conocimientos, los yanesha deben embarcarse en una búsqueda ritual de conocimiento. Estas búsquedas pueden ser breves, simples y con objetivos muy específicos; o pueden ser empresas de larga duración que requieren gran concentración y dedicación personal. Los adultos se embarcan a diario en búsquedas de conocimiento simples. El cazador que masca hojas de coca para 
adivinar qué presa puede cazar, o la mujer que se dispone a cosechar yuca y entona cantos de alabanza a la Paca para que ésta le indique qué plantas tienen los tubérculos más grandes, son ejemplos de este tipo de búsqueda. Estas búsquedas diarias de conocimiento no requieren de gran preparación ritual. Casi todos los adultos yanesha saben cómo adivinar con hojas de coca y muchos de ellos conocen canciones mágicas utilizadas para solicitar la ayuda de una variedad de seres extraordinarios.

La obtención de otros tipos de conocimiento requiere, sin embargo, de búsquedas más prolongadas y un compromiso personal más fuerte. Durante estas búsquedas, generalmente asociadas a rituales de iniciación, los muchachos y muchachas se esfuerzan por aprender dos tipos de conocimiento: el conocimiento administrado por sus padres y parientes cercanos y el conocimiento adquirido mediante sus propias búsquedas personales. Ambos requieren preparación ritual y una guía experimentada. Los muchachos son entrenados en el arte de la cacería por sus padres, abuelos y tíos paternos. Durante su entrenamiento deben respetar un gran número de proscripciones y prescripciones alimenticias. Se les da plantas mágicas epe' (Cyperus sp.) que deben mascar junto con la coca. Mientras las consumen deben permanecer en vigilia. También deben pedirle a Rrera, el águila arpía, dueña de todos los animales y cazador excepcional, y a Pueyomp, el cazador mítico primordial, que los guíe e instruya. Estos seres extraordinarios se presentan a los aprendices -o, más bien, a sus vitalidades-durante las vigilias rituales o los sueños, para otorgarles conocimientos mágicos de cacería o valiosos hechizos de caza.

Los ritos de pubertad femenina también implican una búsqueda ritual de conocimiento. En el pasado, al llegar la menarquia, las muchachas eran recluidas en una choza hecha de hojas de palma hasta por un año. Hoy en día son recluidas por no más de dos meses. Durante este período, la muchacha recluida debe aprender gran parte de los conocimientos que una mujer yanesha debe saber. Reciben lecciones de sus abuelas, madres y tías maternas. Estas les enseñan a hilar algodón, tejer fabricar canastas y esteras, preparar masato, sembrar una chacra y criar niños. E entrenamiento implica no solamente la transmisión de las habilidades físicas necesarias para realizar estas tareas, sino también de los 'secretos,' es decir, los conocimientos extraordinarios necesarios para tener éxito en las mismas. Esto: secretos incluyen canciones para hacer que la yuca y demás cultivos crezcaabundantemente, hierbas para inducir o prevenir el embarazo, y otras plantas mágicas epe' para mantener sanos a los hijos, entrenar a los perros de caza, o retener el amor de la pareja. Para aumentar su capacidad de concentración, las muchachas en reclusión deben de mantenerse en vigilia y silencio ritual. Mascan grande cantidades de hojas de coca y están sujetas a restricciones alimenticias muy estrict: Durante las vigilias rituales, o en sueños, obtienen información importante de boa de los seres extraordinarios. Esta valiosa información se vuelve parte de su cauda personal de conocimiento. 
Las búsquedas de conocimiento relacionadas a rituales de iniciación pueden ser muy exigentes para los iniciados. Sin embargo, las búsquedas más exigentes son aquellas necesarias para conseguir conocimientos especializados. Para convertirse en vegetalista (apartaĩ), chamán tabaquero (pa'llerr), o chamán ayahuasquero, los aprendices deben pasar por un arduo período de entrenamiento bajo la guía de un especialista ya establecido. En el pasado, los sacerdotes yanesha (cornesha') también debían pasar por un entrenamiento semejante. En ambos casos, los iniciados adquirían importantes conocimientos de sus instructores. Pero, al contrario de otros tipos de entrenamiento ritual, el éxito de los chamanes y sacerdotes dependía en gran medida de su capacidad para obtener conocimientos significativos bajo la forma de revelaciones personales. Todos estos especialistas mascaban hojas de coca y practicaban prolongados ayunos y vigilias rituales. Algunos de ellos también consumían narcóticos y sustancias alucinógenas. Según los yanesha, todas estas prácticas inducen a nuestras vitalidades a desprenderse de nuestros cuerpos. Pero mientras que en los sueños, las vitalidades no obedecen por lo general a la voluntad conciente de la persona, cuando se mantiene una vigilia ritual o se consume sustancias tóxicas las vitalidades pueden ser dirigidas concientemente. Esto se logra por medio de la 'fuerza de los pensamientos' del iniciado.

Los yanesha afirman que quienes quieren obtener conocimientos espirituales -ya sea de gente sabia o de seres extraordinarios- deben concentrar su voluntad en aquello que quieren conseguir. Dado que la suya es una cultura oral, el proceso de aprendizaje es concebido como la memorización de pensamientos (yochreta yocsapech). Cuantos más pensamientos se acumulan en el corazón, más fuerte uno se vuelve. Se considera que una persona sabia deriva su poder de la fuerza de sus pensamientos (huomenc poctapñot). Es por medio de este tipo de fuerza que los aprendices de chamán y sacerdote son capaces de dirigir sus vitalidades tal como lo desean.

\section{La vitalidad y sus sentidos}

Las almas/vitalidades tienen los mismos sentidos que los cuerpos que energizan. En contraste con los sentidos corporales, sin embargo, los sentidos incorpóreos de nuestras vitalidades son capaces de percibir la dimensión espiritual de los seres y los objetos de éste y otros niveles del mundo. Los sentidos corpóreos e incorpóreos no son intrínsicamente diferentes; operan de la misma manera y son clasificados en jerarquías de importancia semejantes. Esto no es sorprendente, ya quecomohemos visto es la vitalidad la que confiere a los individuos una vida sensual. El oído es considerado el principal órgano sensorial de nuestra vitalidad. Desde una perspectiva yanesha, el conocimiento espiritual más relevante es aquel obtenido de los seres espirituales mediante revelaciones acústicas, ya sea en sueños o bajo el efecto de alucinógenos. Este también es el caso de otros pueblos amerindios, tales como los wakuénai arahuaco-hablantes (Hill 1993: 214), o los pueblos jíbarohablantes, entre quienes las llamadas "búsquedas de visiones" están más bien 
orientadas hacia la obtención de un mensaje o una profecía de boca del alma arutam de un ancestro poderoso (Taylor 1996: 208-9). En todos estos casos es por medio de la agencia sensorial de la vitalidad de una persona que se adquiere o produce conocimiento significativo.

Los chamanes y sacerdotes yanesha intentan persuadir a los animales, espíritus o divinidades que compartan con ellos sus hechizos, canciones sagradas, profecías u otras formas de locución. Jeñari, un poderoso y renombrado chamán, me contó que durante su entrenamiento su instructor le dijo que debía practicar largas vigilias a fin de obtener la asesoría de un espíritu familiar. Diariamente debía mascar grandes cantidades de hojas de coca mezcladas con jugo de tabaco concentrado. Poco desp comenzó a oír la canción de la zarigüeya y otros animales. Cuando esto sucede, explicó, uno debe de ser extremadamente cuidadoso, ya que puede tratarse de espíritu maligno que está imitando a un animal. Si uno presta atención a la car.c del impostor, el espíritu maligno le roba a uno su vitalidad, causándole la mue Recién después de oír la misma canción diez veces, me dijo, el aprendiz puedecome a repetirla y aprenderla. Esta es la única manera de lograr que el animal dueño canción se convierta en un amigo y protector espiritual (Santos-Granero 2006). I con la adquisición de sus primeros espíritus familiares, los aprendices tam adquieren nuevas entidades incorpóreas. Llamada chañapchenaya, esta alma chamá tiene la capacidad de adoptar la forma de uno de los varios animales protectores chamán. Gracias a estas almas -que solo ellos poseen- los chamanes yanesha tiere capacidad única de transformarse en sus espíritus familiares. Los más importa entre éstos son el jaguar, el picaflor y la anaconda, pero también pueden adoptar formas, tales como aire, viento, o neblina. Encarnados como uno de sus espit familiares, los chamanes no solamente adquieren las capacidades y costumbres animal en cuestión, sino también su 'perspectiva' particular. De esta manera, mier deambulan bajo la forma de jaguar, los chamanes yanesha ven a los seres huma como animales y de esta manera pueden atacarlos.

Los aprendices de sacerdote también buscan obtener revelaciones acú:pero en lugar de dirigirse a los espíritus de animales o plantas, los mismos tratcomunicarse con los dioses creadores- especialmente Yompor Ror (Sol)espíritus mellañoteñ amistosos. Estos seres benéficos solamente se revelar yanesha devotos, es decir, a quienes buscan su protección mediante frect: ayunos, vigilias, rezos, ofrendas, buenos pensamientos y una actitud de sufriente'. Si sus esfuerzos son considerados legítimos, las divinidades manifí su amor/compasión por ellos revelándose por medio de palabras: canc advertencias y otras formas de locución. Los sacerdotes de épocas $\mathrm{F}$ aseguraban que escuchaban las voces de las divinidades en sueños y vigilias ri Estos mensajes podían referirse a la llegada inminente de Yompor Ror, o uno emisarios, a fin de volver a los yanesha inmortales. También podían cons advertencias sobre catástrofes inminentes enviadas por las divinidades como 
por las fallas de sus criaturas humanas. Las revelaciones más apreciadas eran las que incluían canciones sagradas coshaminats. Estas eran reveladas a los sacerdotes más devotos, transmitidas a sus seguidores y cantadas en los centros ceremoniales para alabar a las divinidades y pedir su redención (Smith 1977).

Aunque menos relevante, la vista también es un medio importante a través del cual las vitalidades puede obtener conocimientos significativos. Un aspecto importante del entrenamiento chamánico yanesha es el desarrollo de la capacidad de dirigir nuestras vitalidades tal como uno lo desea. Esta condición es lograda por medio de ayunos, vigilias, y/o el consumo de narcóticos y alucinógenos. Se le enseña a los aprendices a dejar sus cuerpos atrás y a viajar bajo la forma de vitalidades desencarnadas a las regiones escondidas de esta tierra, o a otros niveles del mundo, por encima o por debajo de esta tierra. Los chamanes yanesha aseguran que en estos viajes ven a los animales, espíritus, plantas y objetos "como realmente son"; es decir, pueden ver sus respectivas vitalidades en su forma humana. Bajo este estado los chamanes visitan las montañas donde habitan los 'dueños' de especies animales particulares. También visitan las casas de ciertos seres espirituales, tales como la Madre de la Medicina Vegetal, cuya asesoría es crucial para sus actividades curativas. En otras ocasiones, visitan el mundo sub-acuático, donde conversan con las sirenas, o con los dueños de especies particulares de peces bajo su forma humana. También visitan las cavernas en las que viven las sombras de los difuntos que llevaron una vida correcta y pueden ver algunas enfermedades, tales como la viruela, bajo su forma humana.

La vista no era tan importante como el oído para la búsqueda de conocimientos sacerdotales. Los yanesha aseguran que nadie puede ver a las divinidades. Para verlas, uno debería de practicar una vigilia eternal, lo cual es tanto como decir que uno debería de ser inmortal. Los sacerdotes yanesha también mascaban hojas de coca junto con jugo de tabaco concentrado pero, a diferencia de los chamanes, no realizaban vuelos astrales ni intentaban obtener espíritus familiares. Algunos sacerdotes, sin embargo, eran capaces de detectar o expulsar a los espíritus malignos jo' y los demonios oneñet que amenazaban una determinada región. Y se dice que en ocasiones algunos espíritus mellañoteñ amistosos se dejaban ver por sacerdotes especialmente devotos. Por lo general, sin embargo, los sacerdotes dependían poco de la vista como fuente de conocimiento y daban mayor importancia al oído y a la fuerza de sus pensamientos como modo de aprender y memorizar la información oral. Esto era especialmente cierto en lo relativo al aprendizaje de las canciones sagradas coshamñats, algunas de las cuales tienen hasta setenta y cinco versos (Smith 1977: 282).

Los sentidos de olfato, gusto y tacto de nuestras vitalidades son mucho menos importantes como fuentes de conocimiento. Algunos chamanes me mencionaron las deliciosas fragancias de las mujeres que encuentran durante sus viajes astrales. 
Otros me describieron los ricos olores y sabores de la comida que se les servía durante sus visitas a las moradas de seres extraordinarios. Además, los chamanes yanesha atribuyen algunos malos olores a la presencia de espíritus malignos y demonios y aseguran que ciertas fragancias son clara evidencia de objetos que han sido mágicamente manipulados para actuar como hechizos. Al igual que los muinane (Londoño Sulkin, com. pers.), los yanesha consideran que los olores pueden afectar la subjetividad, en algunos casos enloqueciendo y corrompiendo a las personas, y en otros restaurando su discernimiento moral. Esto es especialmente cierto del hedor de la sangre menstrual, el cual puede debilitar e incluso privar de poderes mágicos tanto a chamanes como a cazadores (ver Belaunde en este volumen). Mediante su capacidad de detectar estos olores, la gente puede tomar las medidas necesarias para mantener a la distancia a los espíritus y personas que pueden causarles daño En términos generales, sin embargo, estos sentidos, en el caso de los yanesha, no juegan un papel tan relevante en la adquisición/producción de conocimiento arcano como el oído y la vista.

\section{Percepción y perspectivismo}

La noción de perspectivismo amerindio (Arhem 1993; Stolze Lima 1999, 200 . 2005; Viveiros de Castro 1998, 2004a y b) ha abierto nuevas y excitantes líneas d: investigación en el campo de los estudios amazónicos. No obstante, ha generad: poca discusión sobre dos aspectos que deberían haber sido centrales al debate: po: un lado, el papel de los diferentes sentidos en las relaciones intra-e inter-específicas (al interior de una misma especie o entre especies diferentes), y por otro, la cuestióde la conciencia tanto en referencia a las capacidades sensoriales de las alma encarnadas y desencarnadas, como en referencia a la naturaleza perspectivista de percepción.

Los estudiosos del perspectivismo no han explorado la dimensión sensorz de este fenómeno excepto para afirmar que los indígenas amazónicos le atribuve: los animales, espíritus y otros seres no-humanos las mismas facultades cognitivas sensoriales que ellos poseen (p.ej. Viveiros de Castro 1998: 474). Se han concentra fundamentalmente en la vista y en como los diferentes tipos de seres 'ven' a otr seres. Uno podría argumentar que la noción de 'perspectiva' es sinónimo de p: de vista y que, por lo tanto, es justificado concentrarse en los aspectos visuales embargo, dado el énfasis en el carácter 'corporizado' de la perspectiva y en la noc de cuerpo como un "haz de afectos y capacidades", uno hubiera esperado perspectiva sobre la perspectiva menos centrada en lo visual. El papel predomin atribuido a la vista respecto de los otros sentidos como el medio privilegiado forjar relaciones entre seres diferentes parece ser, por lo tanto, una proyección nuestra propia jerarquía de los sentidos. Para los yanesha, así como para mu pueblos amazónicos, el oído es una forma de percepción y modo de conocimi más importante que la vista, o que cualquier otro sentido. Esto es cierto en cont 
tanto de relaciones intra- como inter-específicas. El término kayapó mar, que significa simultáneamente 'escuchar' y 'saber', es una expresión de esta difundida concepción (Murphy 2004: 43). Nociones semejantes se encuentran entre los suya, para quienes ku-mba significa 'escuchar-comprender-saber' (Seeger 1981: 83) y entre los pa'ikwené, quienes traducen el término tchimap como 'oír-escuchar-comprender' (Passes 1998: $46 ; 2006)$.

Tampoco se le ha prestado mayor atención a las diferentes capacidades sensoriales de los diversos componentes del ser y, aún más importante, al grado de conciencia que los diferentes tipos de seres tienen sobre estas diferencias. Los investigadores del perspectivismo reconocen la difundida noción 'anímica' según la cual todos los seres están compuestos de un cuerpo y una o más almas, pero asumen que los diferentes seres tienen capacidades sensoriales semejantes. En la versión radical de perspectivismo abogada por Viveiros de Castro todos los seres se ven a sí mismos como humanos mientras que ven a los demás seres como sus opuestos estructurales. "(E)n condiciones normales", se nos dice, "los humanos ven a los humanos como humanos, a los animales como animales y a los espíritus (si es que los ven) como espíritus; sin embargo, los animales (depredadores) y los espíritus ven a los humanos como animales (como presas), en la misma medida en que los animales (como presas) ven a los humanos como espíritus o como animales (depredadores)" (Viveiros de Castro 1998: 470; traducción propia). En circunstancias extraordinarias, sin embargo, las almas de todos los seres se ven a sí mismas, así como a las almas de otros tipos de criaturas, como seres humanos. Esto se encuentra en consonancia con lo que Viveiros de Castro denomina el carácter 'multinaturalista' de las ontologías amerindias, las cuales presuponen la unidad espiritual de todos los seres -en la medida que todos tienen un alma de forma humana- en contraste a su diversidad corporal -en la medida en que las diferentes categorías de seres tienen diferentes tipos de cuerpos. Desde un punto de vista amerindio, la diferencia de perspectiva radicaría, por lo tanto, en el cuerpo.

La presunta unidad espiritual de todos los seres ha sido persuasivamente cuestionada tanto por Rosengren (2006), quien argumenta que las almas no son genéricamente humanas sino que más bien están altamente individualizadas como resultado de condiciones personales, sociales e históricas particulares, y por Londoño Sulkin (2005 y 2006), quien sugiere que las almas y los cuerpos de diferentes especies difieren entre sí de acuerdo a las sustancias de las cuales están hechos, un factor que, a su vez, los hace más o menos morales. En este trabajo quisiera contribuir a esta discusión introduciendo la variable de la conciencia; conciencia de las facultades sensoriales diferenciadas de las vitalidades encarnadas y desencarnadas, pero también de las capacidades sensoriales y cognitivas diferenciadas de los diversos tipos de seres. El perspectivismo radical admite que las almas encarnadas y desencarnadas tienen diferentes habilidades sensoriales: mientras que las primeras se ven a sí mismas como humanas y a los otros tipos de seres como sus opuestos 
estructurales, las segundas se ven a sí mismas y a todas las almas desencarnadas como humanas. Lo que no está claro es si todos los seres son igualmente concientes, primero, de la unidad espiritual de todos los seres, y segundo, de la naturaleza perspectivista de la percepción. Aunque los indígenas amazónicos sostienen que todos los seres humanos se perciben a sí mismos como humanos y a los demás como animales o espíritus, también mantienen que los humanos tienen la capacidad de percibir la dimensión espiritual de todos los seres. Esta habilidad es siempre atribuida a uno de los componentes incorpóreos del ser -yecamquëri entre los yanesha, bedu yushin entre los cashinahua, mekarõ entre los kayapó, likárizva entre los ivakuénai, o wakan entre los jíbaro. Se considera que estos componentes son los únicos dotados de las facultades sensoriales y la agencia necesaria para percibir la dimensión. espiritual del mundo y, por lo tanto, para involucrarse en procesos de adquisición/ producción de conocimiento. Los humanos pueden ver a los animales como animales y a los espíritus como espíritus, pero saben que estos animales y espíritus tienen un alma de forma humana; y saben que pueden interactuar con ellos por medio de la agencia de sus almas o vitalidades.

No está claro, sin embargo, si los animales y espíritus tienen una capacidad de discernimiento semejante. Esta podría ser la mayor diferencia entre los humanos de hoy y los humanos 'primordiales' o 'ex-humanos'. Los humanos son humanos porque saben -es decir, porque poseen la capacidad sensorial incorpórea de saberque la dimensión material del mundo envuelve una dimensión espiritual que tiene una forma diferente, y que la percepción corpórea es siempre perspectivista. Los animales, espíritus y otros seres no-humanos difieren de los humanos en la medida en que no parecen saber esto. Se nos dice que los jaguares ven a los humanos como huanganas y las huanganas ven a los humanos como jaguares; pero saben estos animales que la dimensión espiritual de los animales que ven es humana? Y saben que la percepción perspectivista es multi-direccional? De lo que sabemos hasta ahora, la respuesta es negativa.

Según Stolze Lima (2000: 50; traducción propia), por ejemplo, "La huangana se ve a sí misma como humana, sabe que un juruna es un semejante, pero no sabe que es una huangana para un juruna." Lo que distingue a los humanos de los animales o de los espíritus es, ante todo, que los humanos son concientes de la naturaleza perspectivista de la percepción, mientras que los animales y los espíritus no lo son. Esto muy bien podría derivar del hecho de que los animales y espíritus están constituidos de manera diferente -ya sea porque carecen de almas o de cuerpos verdaderos- $y$, por lo tanto, tienen diferentes modos de sentir y conocer. En muchas sociedades amerindias se considera que los animales no tienen verdaderas almas individuales (Viveiros de Castro 1998: 471). Más bien, se piensa que los animales son la manifestación individual del alma primordial de la especie, a menudo conocida como 'jefe', 'dueño' o 'madre' de la especie. A su vez, a los espíritus les falta un cuerpo verdadero. Tienen forma corporal y, tal como lo señala Viveiros de Castro 
(1998: 481), no son completamente inmateriales, pero no se les atribuye el tipo de vida corporal y sensual característica de los humanos. Privados de verdaderas almas verdaderos cuerpos, estos seres están dotados de perspectiva, pero no son concientes de que otros seres están igualmente dotados. Es esta capacidad-conciencia de la naturaleza perspectivista de la percepción- y conocimiento -conciencia de la esencia humana de todos los seres- que los animales y espíritus perdieron o no adquirieron cuando fueron transformados como tales al final de los tiempos míticos.

Por esta razón, no puede afirmarse que los indígenas amazónicos piensan que los animales son humanos. Más bien, lo que afirman, tal como lo expresa hábilmente Stolze Lima (1999: 113), es que "los animales piensan que son humanos." Esto pone en cuestión la versión radical del perspectivismo, la cual implica que todas las perspectivas tienen igual valor (Arhem 1993: 124). Ello no implica, sin embargo, lo opuesto; es decir, la existencia de una perspectiva absoluta. Los humanos, animales y espíritus pueden imponer sus perspectivas unos a otros, pero el resultado de este intercambio de perspectivas, tal como sugiere Stolze Lima (2000: 48), no puede ser determinado a priori. Más bien, el hecho de que los animales "piensen que son humanos" pero no sean concientes de que los humanos son esencialmente humanos, indica que en las cosmologías amerindias la conciencia de la naturaleza perspectivista de la percepción es solamente atribuida a los humanos actuales. Esto, a su vez, sugiere dos cosas. Primero, que los pueblos amerindios pueden tener visiones del mundo mucho más antropocéntricas -si por el prefijo 'antro' entendemos a los humanos de hoy en día en contraposición a los humanos de la era primordialque lo que estamos preparados a aceptar (Viveiros de Castro 1998: 477). ${ }^{2} Y$, segundo, que en vez de abogar por un único y monolítico tipo de perspectivismo amerindio, sería mejor hablar de una variedad de 'cosmologías perspectivistas' en las que los elementos centrales del perspectivismo son combinados en diferentes permutaciones y con diferentes pesos relativos (Londoño Sulkin 2005: 24; pero también Stolze Lima 2000: Nota 6). Desde el punto de vista de estas formas menos radicales de perspectivismo, es la ilusión en la que están inmersos los animales -ilusión que los hace pensar que son humanos- que hace que la vida humana sea tan peligrosa (Stolze Lima 1999: 113). Este peligro es aún mayor como resultado de las deficiencias que los indígenas amazónicos le atribuyen a las facultades sensoriales tanto corpóreas como incorpóreas.

Nótese, por ejemplo, que la mayor parte de los relatos perspectivistas amerindios se concentran en la forma en que los humanos ven a los animales y espíritus o en la forma en que diferentes tipos de animales y espíritus ven a los humanos. Poco se dice sobre cómo los animales carnívoros ven a los espíritus o cómo los animales herbívoros ven a los animales carnívoros. Puede que las cosmologías amerindias sean 'multinaturalistas' pero continúan estando sustentadas en la centralidad y primacía de los seres humanos, o más precisamente, de los seres humanos actuales. 


\section{Deficiencias Perceptuales}

Las diferencias que los yanesha atribuyen a las vitalidades encarnadas y desencarnadas en términos de sus capacidades sensoriales darían lugar a un sistema altamente organizado de conocimiento y percepción si no fuera por dos problemas. En primer lugar, desde la perspectiva yanesha, ni los sentidos corpóreos ni los incorpóreos son infalibles. Ambos son imperfectos y deficientes y, por lo tanto, susceptibles a cometer errores. En segundo lugar, las dimensiones materiales y espirituales del mundo no son ni auto-contenidas ni están estrictamente separadas. Pueden existir en una suerte de forma paralela, pero en la medida que no existen discontinuidades entre la una y la otra, constituyen una misma realidad. Debido a esta cualidad, con frecuencia las vitalidades encarnadas y desencarnadas de los diversos seres entran en contacto en maneras que transgreden los medios normales de percepción, es decir, los medios de percepción de la vida corpórea y despierta. Ambas situaciones -falla perceptual y transgresión perceptual-implican graves peligros para los seres involucrados en estas relaciones.

Los yanesha son cuidadosos al indicar que la percepción sensual -ya sea corpórea o incorpórea- puede ser engañosa. Existen muchas instancias en que los sentidos corporales engañan a la persona durante sus actividades diarias. Los Yanesha consideran, sin embargo, que los sentidos de sus almas/vitalidades también son imperfectos. Cuentan, por ejemplo, que cuando visitan la morada sobrenatural de Shemellama yarr, el Jaguar-Avispa que es 'dueño' de la variedad de tabaco de flor roja usada por los chamanes hechiceros, las almas de estos jaguares bajo su forma humana le ofrecen al aprendiz de chamán un trozo de carne ahumada o asada que parece ser carne de monte, pero que en realidad es carne humana. Si el aprendiz ingenuo o descuidado come la carne que le es ofrecida, desarrolla el deseo de come: carne humana. De ahí en adelante su alma chañapchenaya se transforma en jaguar antropófago o comedor-de-gente cada vez que siente deseos de comer carne.

Los encuentros con seres espirituales durante vuelos astrales también pueden ser engañosos. Cuando la vitalidad de un chamán visita otros niveles del mundo sus sentidos sólo pueden percibir las almas de forma humana de sus habitantes; ra pueden detectar sus cuerpos no-humanos tal como los verían estando despiertos De esta manera, durante sus vuelos, los chamanes no pueden distinguir entre los diferentes seres que encuentran (ver Vilaça 1992: 82, para una noción War: semejante). Hermosas mujeres de largos cabellos, ataviadas con túnicas brillantes pintadas con intrincados diseños, y adornadas con collares de semillas fragant: pueden ser una mujer jaguar, una sirena, la 'madre' del ayahuasca, la hija del Tạ: primordial, o un espíritu mellañoteñ femenino. Con el paso del tiempo, los chamanaprenden a distinguir una manifestación de la otra por sus adornos particularmente, por los diseños de sus túnicas. Pero durante el período entrenamiento su inexperiencia puede llevarlos a tener relaciones sexuales con algu- 
de estas mujeres. Si la mujer en cuestión es un espíritu benéfico, el encuentro puede ser no sólo placentero sino altamente beneficioso; el aprendiz puede obtener de ella conocimientos extraordinarios de gran valor. Pero si la mujer es un espíritu maligno o una mujer comedora-de-gente, entonces la misma puede robarle al aprendiz su vitalidad, causándole de esta manera su muerte.

La transgresión perceptual es igualmente riesgosa. Percibir con los sentidos del cuerpo el alma/vitalidad de otro ser -la cual normalmente sólo puede ser percibida con los sentidos incorpóreos- es siempre un acto peligroso. Este fue el caso cuando escuché el canto de Pocoy, tal como cuento en la narración con la que di comienzo a este artículo. Y ello explica por qué Matar estaba tan ansioso por alejarme del río lo más rápido posible. Cuando un pescador yanesha desaparece y su cuerpo nunca es encontrado, su desaparición es casi siempre atribuida a las acciones seductoras de las sirenas. Los cazadores yanesha también son susceptibles de tener este tipo de encuentros, aunque en su caso suelen encontrarse con las vitalidades de los animales de caza o con las formas primordiales de las diferentes especies animales. Estos encuentros siempre son peligrosos en la medida que implican el pasaje hacia un espacio-tiempo liminal en el que las vitalidades encarnadas de los humanos entran en contacto con las vitalidades desencarnadas de los animales. El Tapir primordial puede revelarse a un cazador que ha herido y abandonado a uno de 'sus' tapires, a fin de castigarlo por su falta de cuidado robándole su vitalidad. En otras circunstancias, la vitalidad de una huangana puede presentarse ante un cazador perdido bajo la forma de una bella mujer resuelta a seducirlo y llevarlo a vivir con ella en su morada sobrenatural (ver Rosengren 2006, sobre encuentros liminales semejantes). En estos casos, el cazador adopta el punto de vista -la perspectiva- de la huangana y con ello su forma animal (Viveiros de Castro 1998: 483). De esta manera, evitar que los sentidos corporales perciban las vitalidades desencarnadas de seres diferentes de uno mismo es tan importante como entrenar a los sentidos incorpóreos a identificar las almas de forma humana que uno encuentra en sueños con los cuerpos a los que éstas realmente pertenecen.

* $\quad * \quad *$

Las maneras yanesha de sentir y conocer son muy diferentes de las nuestras. Aunque existen importantes semejanzas entre su teoría de la percepción y aquella desarrollada por Platón, esta última difiere de manera sustancial, particularmente porque carece de la dimensión perspectivista tan típica de las cosmologías amerindias. Desde un punto de vista yanesha, el conocimiento significativo es siempre un conocimiento extraordinario; es decir, un conocimiento de y sobre la dimensión espiritual del mundo vivido. Este tipo de conocimiento no puede ser obtenido por medio de nuestros sentidos corporales; sólo puede ser adquirido por medio de la agencia conciente de uno de los componentes incorpóreos del ser. Ya sea obtenido directamente, o aprendido de otros individuos, el conocimiento 
significativo siempre se origina a través de modos de sentir y conocer incorpóreos. En las sociedades amerindias el conocimiento siempre se 'encarna' o 'hace cuerpo', tal como Belaunde (2005) sostiene persuasivamente en su discusión sobre la hematología amazónica, pero el cuerpo es el sitio de conocimiento, no su agente causante.

Es por medio del conocimiento adquirido de seres que habitan otras dimensiones o niveles del mundo que los indígenas amazónicos han aprendido cóm: hacer y re-hacer los cuerpos humanos. Esto por lo general sostienen que los rituales de iniciación -los cuales siempre implican algún grado de transformación de los cuerpos de los iniciados- les fueron enseñados por seres extraordinarios, tales comLuna, Águila Arpía, Jaguar y otros. Los cuerpos de los seres humanos son moldeados y modelados a lo largo de una serie de ritos de pasaje a través del conocimier: obtenido de estos seres. La adquisición de nuevos conocimientos también genera importantes transformaciones corporales. Los pueblos de habla jibaro aseguran çlos hombres y mujeres que han obtenido una revelación arutam adquieren una may confianza en sí mismos, confianza que se manifiesta en la postura y movimiento de sus cuerpos. Aquellas personas que no han obtenido una revelación pueden ser identificadas porque no tienen ni una postura orgullosa ni un aire de mando. I yanesha atribuyen el mismo tipo de transformación corporal a los chamanes sacerdotes que han tenido éxito en adquirir una revelación acústica -sea un mensz o una canción- de uno de los tantos animales, espíritus y divinidades. Incluso cuerpos de los muertos son transformados mediante el conocimiento ritual, tal com Caiuby (2006) demuestra de manera tan convincente en su discusión sobre procesos de des-figuramiento y re-figuramiento que tienen lugar en los rituak= funerarios bororo. Por esta razón, sugiero que, desde una perspectiva amerindia, vez de ser la causa de conocimiento, los cuerpos son causados por conocimiento; conocimiento que sólo puede ser adquirido a través de sus almas sensuales.

\section{Agradecimientos}

Un primer borrador de este artículo fue presentado en la "Conferencia de estudiantes en aprex de su profesora Joanna Overing", que organizamos George Mentore y yo, y que tuvo lugar en: Universidad de Virginia los días 28-29 de noviembre de 2005. Quisiera agradecer a George Mentore el provechoso diálogo que mantuvimos sobre los modos amerindios de conocimiento durante las etą̧: preparatorias de la conferencia. También quisiera agradecer a Carlos Londoño Sulkin y a Stes Rubenstein por sus comentarios sobre el primer borrador de este artículo. Debo, además, un espeagradecimiento a Luisa Elvira Belaunde por haberse tomado el trabajo de traducir este artículo inglés al español. 


\section{BIBLIOGRAFÍA}

Arhem, Kaj

1993 Ecosofía makuna. La selva humanizada: ecología alternativa en el trópico húmedo colombiano. François Correa, editor, pp. 109-126. Bogotá: ICA/FEN/CEREC.

Belaunde, Luisa Elvira

2005 El recuerdo de Luna: Genero, sangre y memoria entre los pueblos amazónicos. Lima: UNMSM.

Caiuby Novaes, Sylvia

2006 Bororo Funerals. Images of the Refacement of the World. Special Issue in Honor of Professor Joanna Overing. Tipiti (en prensa)

Classen, Constance

2005 McLuhan in the Rainforest. The Sensory Worlds of Oral Cultures. Empire of the Senses. The Sensual Culture Reader. David Howes, editor, pp. 147-163. Oxford \& New York Berg.

Crocker, Jon Christopher

1985 Vital Souls. Bororo Cosmology, Natural Symbolism, and Shamanism. Tucson: The University of Arizona Press.

Descola, Philippe

1996 The Spears of Twilight. Life and Death in the Amazon Jungle. Glasgow: HarperCollins Publishers.

Gow, Peter

2006 Purús Song. Nationalization and Tribalization in Southwestern Amazonia.» Special Issue in Honor of Professor Joanna Overing. Tipiti (en prensa)

Hill, Jonathan D.

1993 Keepers of the Sacred Chants. The Poetics and Ritual Pozver in an Amazonian Society. Tucson \& London: The University of Arizona Press.

Howes, David (ed.)

1991 The Varieties of Sensory Experience. University of Toronto Press.

2005 Empire of the Senses. The Sensual Culture Reader. Oxford \& New York: Berg.

Kensinger, Kenneth

1995 How Real People Ought to Live. The Cashinahua of Eastern Peru. Prospect Heights, IL: Waveland Press. 
Lagrou, Elsje Maria

2000 Homesickness and the Cashinahua Self: A Reflection on the Embodied Condition of Relatedness. The Anthropology of Love and Anger. The Aesthetics of Conviviality in Native Amazonia. Joanna Overing and Alan Passes, editores, pp. 152-169. London \& New York: Routledge.

Lima, Tânia Stolze

1999 The Two and its Many. Reflections on Perspectivism in a Tupi Cosmology. Ethnos 64(1):107-31.

2000 Towards an Ethnographic Theory of the Nature/Culture Distinction in Juruna Cosmology. Brazilian Review of Social Sciences 1:43-52.

2005 Um peixe olhou para mim. O povo Yudja e a perspectiva. São Paulo: UNESP.

Londoño Sulkin, Carlos

2005 Inhuman Beings: Morality and Perspectivism among Muinane People (Colombian Amazon). Ethnos 70(1): 7-30.

2006 «Instrumental Speeches, Morality, and Masculine Agency among Muinane People (Colombian Amazon)." Special Issue in Honor of Professor Joanna Overing. Tipiti (en prensa)

McLuhan, Marshall

1961 Inside the Five Sense Sensorium. The Canadian Architect 6:49-54.

Murphy, Isabel

2004 And I, In My Turn, Will Pass It On. Knowledge Transmission Among the Kayapó. Dallas, TX: SIL International.

Overing, Joanna

1975 The Piaron, A People of the Orinoco Basin. A Study in Kinship and Marriage. Oxford: Clarendon Press.

1985 Reason and Morality. London \& New York: Tavistock Publications.

2006 The Stench of Death and the Aromas of Life: Poetics of Ways of Knowing and Sensory Process among Piaroa of the Orinoco Basin. Special Issue in Honor of Professor Joanna Overing. Tipiti (en prensa)

Passes, Alan

1998 The Hearer, the Hunter, and the Agouti Head: Aspects of Intercommunication and Conviviality Among the Pa'ikwené (Palikur) of French Guiana. Ph.D. Dissertation, University of St Andrews.

2001 Hearing as Understanding: The Value of Good Listening in Native Amazonia. Paper for Queen's University, Belfast, 9 October 2001.

2006 From One to Metaphor: Toward an Understanding of Pa'ikwené (Palikur Mathematics. Special Issue in Honor of Professor Joanna Overing. Tipiti (en. prensa) 
Rosengren, Dan

2006 Matsigenka Corporeality: A Non-Biological Reality. On Notions of Consciousness and the Constitution of Identity. Special Issue in Honor of Professor Joanna Overing. Tipiti (en prensa)

Santos-Granero, Fernando

1991 The Powver of Love: The Moral Use of Knowledge amongst the Amuesha of Central Peru. London: Athlone Press.

2003 Pedro Casanto's Nightmares: Lucid Dreaming in Amazonia and the New Age World. Tipiti. Journal of the Society for the Anthropology of Lowland South America 1(2):179-210.

2006 Of Fear and Friendship: Amazonian Sociality beyond Kinship and Affinity. Journal of the Royal Anthropological Institute. (en prensa)

Seeger, Anthony

1981 Nature and Society in Central Brazil. The Suya Indians of Mato Grosso. Cambridge, MA: Harvard University Press.

Shepard Jr, Glenn H.

2004 A Sensory Ecology of Medicinal Plant Therapy in Two Amazonian Societies. American Anthropologist 106(2):252-266.

Smith, Richard Chase

1977 Deliverance from Chaos for a Song: A Social and Religious Interpretation of the Ritual Performance of Amuesha Music. Ph.D. Dissertation, Cornell University.

Taylor, Anne Christine

1996 The Soul's Body and its States: An Amazonian Perspective on the Nature of Being Human. Journal of the Royal Anthropological Institute 2:201-15.

Vilaça, Aparecida

1992 Comendo como gente: formas do canibalismo wari (Pakan Nova). Rio de Janeiro: Editora UFRJ.

Viveiros de Castro, Eduardo

1998 Cosmological Deixis and Amerindian Perspectivism. Journal of the Royal Anthropological Institute 4(3):469-488.

2004a Perspectival Anthropology and the Method of Controlled Equivocation. Tipiti 2(1): 3-22.

2004b Exchanging Perspectives: The Transformation of Objects into Subjects in Amerindian Ontologies. Common Knowledge 10(3): 463-484.

Wittgenstein, Ludwig

1974 Philosophical Investigations. Oxford: Blackwell. 